\title{
The fast debris evolution model
}

\author{
H.G. Lewis *, G.G. Swinerd, R.J. Newland, A. Saunders \\ Astronautics Research Group, School of Engineering Sciences, University of Southampton, Highfield, Southampton SO17 1BJ, United Kingdom
}

Received 11 October 2008; received in revised form 20 May 2009; accepted 22 May 2009

\begin{abstract}
The 'particles-in-a-box' (PIB) model introduced by Talent [Talent, D.L. Analytic model for orbital debris environmental management. J. Spacecraft Rocket, 29 (4), 508-513, 1992.] removed the need for computer-intensive Monte Carlo simulation to predict the gross characteristics of an evolving debris environment. The PIB model was described using a differential equation that allows the stability of the low Earth orbit (LEO) environment to be tested by a straightforward analysis of the equation's coefficients. As part of an ongoing research effort to investigate more efficient approaches to evolutionary modelling and to develop a suite of educational tools, a new PIB model has been developed. The model, entitled Fast Debris Evolution (FADE), employs a first-order differential equation to describe the rate at which new objects $\geqslant 10 \mathrm{~cm}$ are added and removed from the environment. Whilst Talent [Talent, D.L. Analytic model for orbital debris environmental management. J. Spacecraft Rocket, 29 (4), 508-513, 1992.] based the collision theory for the PIB approach on collisions between gas particles and adopted specific values for the parameters of the model from a number of references, the form and coefficients of the FADE model equations can be inferred from the outputs of future projections produced by high-fidelity models, such as the DAMAGE model.

The FADE model has been implemented as a client-side, web-based service using JavaScript embedded within a HTML document. Due to the simple nature of the algorithm, FADE can deliver the results of future projections immediately in a graphical format, with complete user-control over key simulation parameters. Historical and future projections for the $\geqslant 10 \mathrm{~cm}$ LEO debris environment under a variety of different scenarios are possible, including business as usual, no future launches, post-mission disposal and remediation. A selection of results is presented with comparisons with predictions made using the DAMAGE environment model. The results demonstrate that the FADE model is able to capture comparable time-series of collisions and number of objects as predicted by DAMAGE in several scenarios. Further, and perhaps more importantly, its speed and flexibility allows the user to explore and understand the evolution of the space debris environment.
\end{abstract}

(C) 2009 COSPAR. Published by Elsevier Ltd. All rights reserved.

Keywords: Space debris; Modelling; Particles-in-a-box

\section{Introduction}

The exploitation of near-Earth space has resulted in the persistent generation of space debris such that operational spacecraft make up less than $8 \%$ of the $\sim 13,000$ current catalogued orbiting objects. In addition, fragmentation debris generated by spontaneous disintegration or by accidental or deliberate collisions account for $50 \%$ of all orbital debris, with this figure expected to rise in the future as acci-

\footnotetext{
${ }^{*}$ Corresponding author.

E-mail address: hglewis@soton.ac.uk (H.G. Lewis).
}

dental collisions involving large, intact objects are predicted to become the primary debris-generating mechanism even in the absence of future space launches (Liou and Johnson, 2006; Krisko, 2007). Whilst the international community have been working towards the development and implementation of key debris mitigation guidelines, these alone may not be sufficient to stabilise the low Earth orbit (LEO) debris environment and other methods, such as active debris removal (Liou and Johnson, 2007), may become necessary.

Predictions of the future debris environment can be made using computer codes that incorporate the main sources and sinks of orbital debris with a propagator that 


\section{Nomenclature}

\begin{tabular}{|c|c|}
\hline$A$ & deposition coefficient (from Talent, 1992) \\
\hline$a$ & atmospheric decay rate coefficient \\
\hline$B$ & removal coefficient (from Talent, 1992) \\
\hline$C$ & collision coefficient (from Talent, 1992) \\
\hline$\dot{C}$ & collision rate $\left(\# \mathrm{yr}^{-1}\right)$ \\
\hline$D$ & general atmospheric decay parameter \\
\hline$\dot{D}$ & $\begin{array}{l}\text { rate of change of the atmospheric decay param- } \\
\text { eter }\left(\% \mathrm{yr}^{-1}\right)\end{array}$ \\
\hline$\dot{E}$ & explosion rate $\left(\# \mathrm{yr}^{-1}\right)$ \\
\hline$f_{c_{1}}$ & $\begin{array}{l}\text { fraction of collisions that are catastrophic } \\
(>40 \mathrm{~J} / \mathrm{g})\end{array}$ \\
\hline$f_{c_{2}}$ & $\begin{array}{l}\text { fraction of collisions that are damaging }(<40 \mathrm{~J} / \\
\mathrm{g})\end{array}$ \\
\hline$k_{1}, k_{2}$, &.$k_{6}$ collision rate coefficients \\
\hline & launch rate $\left(\# \mathrm{yr}^{-1}\right)$ \\
\hline$\dot{M}$ & $\begin{array}{l}\text { combined mitigation and direct removal rate } \\
\left(\# \mathrm{yr}^{-1}\right)\end{array}$ \\
\hline$N$ & number of objects on orbit (from Talent, 1992) \\
\hline$N_{c}$ & number of collision fragments on orbit \\
\hline$N_{E}$ & number of explosion fragments on orbit \\
\hline$N_{I}$ & $\begin{array}{l}\text { number of intact objects (payloads and upper } \\
\text { stages) on orbit }\end{array}$ \\
\hline
\end{tabular}

$N_{k} \quad$ threshold total number of objects on orbit

$N_{p} \quad$ number of objects of a particular type on orbit

$N_{T} \quad$ total number of objects on orbit

$n_{c_{1}} \quad$ number of fragments produced per catastrophic collision

$n_{c_{2}} \quad$ number of fragments produced per damaging collision

$n_{E} \quad$ number of fragments produced per explosion

$n_{L} \quad$ number of intact objects added to environment per launch number of objects added to environment by a typical event

$\dot{P} \quad$ typical event rate

$R_{c} \quad$ collision fragment 'radius'(m)

$R_{E} \quad$ explosion fragment 'radius' (m)

$R_{I} \quad$ intact object 'radius' (m)

$R_{p} \quad$ typical object 'radius' (m)

$t \quad$ current simulation time (years)

$\Delta t \quad$ time-step (years)

evolves the population in three dimensions over time. Traditionally, the data that are reported by these codes includes the time-history of the number of objects on orbit and the number of collisions. Outputs may also include collision rates for different orbits, object sizes and object types, amongst others, although this information is typically not used when assessing the impact of debris prevention practices, with preference given to measures of environmental stability, such as the rate of change of the number of objects on orbit. Due to the uncertainty in future space activities and the probabilistic nature of the algorithms making up the codes, reliable statistics are only derived from tens or even hundreds of separate simulations using Monte Carlo (MC) methods (Liou, 2005). In particular, collisions provide the greatest source of variability between simulations; in a study of the historical evolution of the LEO debris environment (Fig. 1), the intermittent, random bursts of collisional activity result in individual simulations where no two outcomes are alike, even when adopting the same, historical launch activity. Thus, the process of predicting the future debris environment using these codes is a lengthy, computationally expensive task.

Simpler methods for simulating the LEO debris environment have been investigated, including the 'particles-in-abox' (PIB) approach (Farinella and Cordelli, 1991; Talent, 1992), but these have been largely superseded by the complex computer codes and the MC modelling approach described above in an attempt to increase the knowledge that can be extracted from computer models of the debris environment. The PIB method uses a differential equation to describe the rate of change of the number of objects in LEO,

$\dot{N}=A+B N+C N^{2}$,

with coefficients $A, B$ and $C$ that are linked to physical properties of the LEO environment, and roots that describe characteristic behaviour (Talent, 1992). There is very little computational cost associated with making predictions of the future LEO debris environment with this approach and, as such, it can be used to assess a variety of scenarios quickly. Whilst the method is limited to a small set of states that can be analysed (number of objects, total mass and average object radius) there is considerable overlap between these and the time-histories typically reported by the more complex, three-dimensional computer codes when the latter are used for assessing debris prevention strategies. In this case, the predictions made by both methods tend to show good agreement. In addition, and from an educational standpoint, the simple, differential form of the model provides a useful mechanism for explaining the evolution of the debris environment to students, the media and the general public, thereby enhancing the overall awareness of the debris problem.

The intention of this paper, therefore, is to present the formulation of a new PIB approach, implemented as a real-time, internet-based service, and to assess the performance of the method on a variety of future scenarios by comparing its outputs to those produced by a three-dimensional evolutionary model. The new approach, entitled Fast Debris Evolution (FADE), adopts a differential 


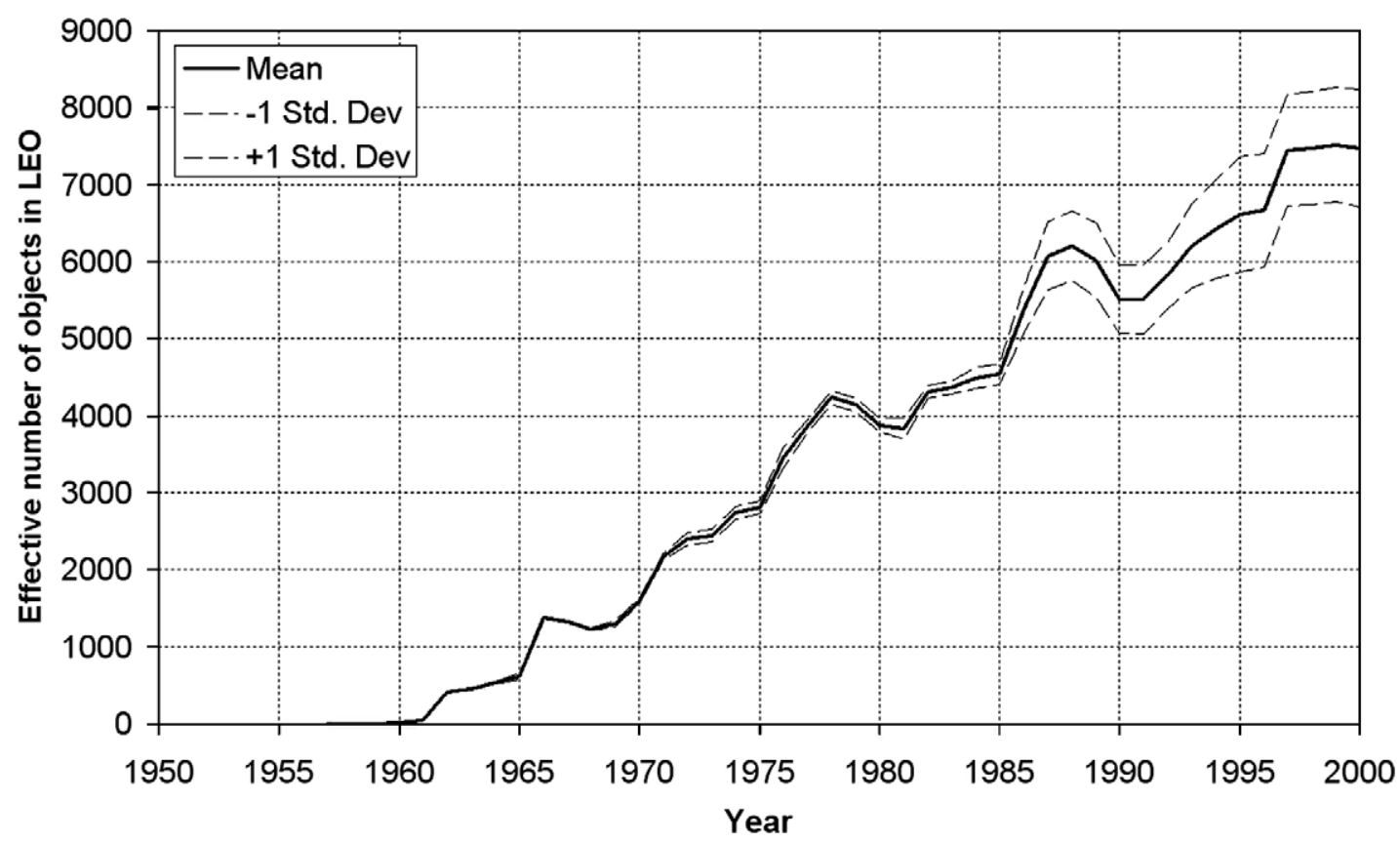

Fig. 1. The effective number of objects in low Earth orbit (LEO) for the historical period up to 1 January 2000 as simulated by DAMAGE: the average and standard deviation of 40 individual Monte Carlo (MC) simulations.

equation 'engine' in a manner similar to Talent's PIB method but uses the outcome of a MC simulation performed by the three-dimensional model to identify appropriate differential coefficients. In this way, it was anticipated that the predictions made by the new FADE method would prove to be useful from both a scientific and educational perspective.

\section{Method}

The PIB method described by Talent (1992) makes the assumption that the debris population moves randomly and has access to the entire LEO volume in order to define the collision coefficient, $C$, in Eq. (1). An 'incomplete mixing factor', related to the eccentricities of objects on orbit, is employed to moderate the effect of this assumption but it is not obvious how this may be defined although it is clear from Eq. (1) that this parameter has a significant effect on the response of the PIB model. The FADE approach makes a similar assumption about the motion of objects within the LEO volume but uses an analysis of collision rates produced by the Debris Analysis and Monitoring Architecture for the Geosynchronous Environment (DAMAGE) model to identify appropriate coefficients for the differential equation.

The University of Southampton's debris model, DAMAGE, is a three-dimensional model that was initially aimed at simulating debris within the geosynchronous orbital regime but has since been upgraded to allow investigations of the full LEO-to-GEO debris environment. As with other evolutionary models, DAMAGE is able to simulate the historical and future debris populations $\geqslant 10 \mathrm{~cm}$ using a
Monte Carlo approach, whereby multiple projection runs are performed to establish reliable statistics on the outcome. Projections covering the historical period from 1957 to 2000 (for this paper) employ launch and fragmentation information from ESA's Database and Information System Characterising Objects In Space (DISCOS) and historical monthly averaged solar flux F10.7 values combined with the CIRA-72 atmospheric model for atmospheric drag calculation. Future projections covering periods from the beginning of 2000 (in this paper) use statistics derived from DISCOS data covering the period 1996-2001 to simulate future launch and explosion activity, with a long-term F10.7 projection based on a repeating sine function. The historical and future fragmentation events are simulated using the NASA Standard Breakup Model (Johnson et al., 2001) and non-fragmentation sources of debris, except mission-related objects included in DISCOS, are not considered. All objects are propagated forwards using a semi-analytical orbital propagator that includes Earth's $J_{2}, J_{3}, J_{2,2}$, luni-solar gravitational perturbations, solar radiation pressure (with cylindrical Earth shadow) and atmospheric drag. Collision probabilities are estimated using a fast, pair-wise algorithm based on the 'Cube' approach adopted in NASA's LEO-to-GEO Environment Debris model (LEGEND; Liou et al., 2004).

In efforts to validate the algorithms employed within DAMAGE, the model has been used to estimate collision rates between Jovian satellites, and orbital debris $\geqslant 10 \mathrm{~cm}$ in near-Earth space over historical and future periods. The DAMAGE model shows good agreement with results reported by Kessler (1981) and Liou et al. (2003) for collision rates between the moons of Jupiter (Table 1), but the 
Table 1

Collision rates $\left(10^{-10} \mathrm{yr}^{-1}\right)$ between Jovian satellites.

\begin{tabular}{llll}
\hline Collision pair & Kessler (1981) & Liou et al. (2003) & DAMAGE \\
\hline Himalia-Elara & 4.3 & 4.2 & 4.1 \\
Himalia-Lysithea & 2.8 & 3.5 & 3.3 \\
Himalia-Leda & 3.1 & 3.2 & 3.0 \\
Elara-Lysithea & 0.52 & 0.52 & 0.51 \\
Elara-Leda & 0.57 & 0.56 & 0.65 \\
Lysithea-Leda & 0.039 & 0.038 & 0.033 \\
\hline
\end{tabular}

Table 2

Average number of catastrophic collisions, with standard deviations, for historical and combined historical-future projection periods (based on a 'business as usual' scenario).

\begin{tabular}{lll}
\hline Projection period & Krisko $(2007)^{\mathrm{a}}$ & DAMAGE \\
\hline $1957-2006(50$ years $)$ & $0.9( \pm 1.0)$ & $1.5( \pm 1.1)$ \\
$1957-2035(79$ years $)$ & $3.3( \pm 2.0)$ & $7.4( \pm 2.6)$ \\
\hline
\end{tabular}

${ }^{\text {a }}$ Target and impactor $\geqslant 10 \mathrm{~cm}$.

results differ from those reported by Krisko (2007) for the near-Earth debris environment future component (Table 2). The differences likely arise from the use of different traffic and explosion assumptions for the future component, and modifications made to the pair-wise collision algorithm in DAMAGE, which were introduced to recover instances where objects are approximately co-located but are placed into adjacent 'cubes' by the original algorithm.

\subsection{The FADE approach}

Following the approach adopted by Talent (1992), the number of objects $\geqslant 10 \mathrm{~cm}$ resident in the LEO environment at any given time was selected as the 'state' quantity. This parameter is recorded by DAMAGE as a matter of routine and thus provides a useful measure for comparison purposes. However, in a departure from Talent's model, the FADE approach recognises the contribution to the environment of three fundamental debris types; intact objects (including payloads and upper stages), explosion fragments and collision fragments. Hence, the differential equation used in FADE takes the form,

$\dot{N}_{T}(t)=\dot{N}_{I}(t)+\dot{N}_{E}(t)+\dot{N}_{C}(t)$.

Given initial values for $N_{I}, N_{E}$, and $N_{C}$, at time $t_{0}$, future values are estimated using Euler's Method. For example, for intact objects we have

$N_{I}(t+\Delta t)=N_{I}(t)+\dot{N}_{I}(t) \Delta t$.

Whilst more accurate differential equation solvers exist, it was felt that Euler's Method was appropriate given the relatively small values used for the timestep, $\Delta t$, (typically one year), and the need to keep the algorithm fast and transparent for educational purposes. The total number of objects in the LEO volume at time $t$ can then be determined from,

$N_{T}(t)=N_{I}(t)+N_{E}(t)+N_{C}(t)$.
The rate of change of the number of intact objects is,

$\dot{N}_{I}=\dot{L} n_{L}-\frac{D}{R_{I}} N_{I}-\dot{E}-\left(1+f_{C_{1}}\right) \dot{C}-\dot{M}$,

(for clarity the notation indicating time dependence has been dropped here, and below), the rate of change of the number of explosion fragments is,

$\dot{N}_{E}=\dot{E} n_{E}-\frac{D}{R_{E}} N_{E}$,

and the rate of change of the number of collision fragments is,

$\dot{N}_{C}=\dot{C}\left(f_{C_{1}} n_{C_{1}}+f_{C_{2}} n_{C_{2}}\right)-\frac{D}{R_{C}} N_{C}-f_{C_{2}} \dot{C}$.

In Eqs. (5)-(7) there is both a 'deposition' term of the form, $\dot{P} n_{P}$,

which describes the number of objects of a particular type, $n_{P}$, added to the environment by events that occur with yearly rate, $\dot{P}$, and a 'removal' term of the form,

$-\frac{D}{R_{P}} N_{P}$

which follows, in part, Talent's (1992) method for describing natural decay due to the residual atmosphere in LEO. In FADE, however, it is assumed that the average radius of each object type remains constant. This assumption was found to be reasonable, following an analysis of the time-history of the average radii of intact objects, explosion fragments and collision fragments in LEO for historical and future evolutions with DAMAGE, which showed only minor variations in these values. Other 'removal' mechanisms are included in Eq. (5) (for intact objects) and Eq. (7) (for collision fragments). In both cases, the underlying assumption is that collisions are either intact-intact (catastrophic collisions, where the ratio of projectile kinetic energy to target mass exceeds $40 \mathrm{~J} / \mathrm{g}$ ) or intact-fragment (damaging collisions, $<40 \mathrm{~J} / \mathrm{g}$ ), and objects are removed accordingly. In addition, each explosion removes one intact object whilst mitigation, direct retrieval or active debris removal reduces the population of intact objects at a rate $\dot{M}$ per year.

The launch rate, $\dot{L}$, and the explosion rate, $\dot{E}$, are constant, whilst the collision rate is described by combining two quadratic equations,

$\dot{C}= \begin{cases}k_{1}+k_{2} N_{T}+k_{3} N_{T}^{2}, & N_{T}<N_{k}, \\ k_{4}+k_{5} N_{T}+k_{6} N_{T}^{2}, & N_{T} \geqslant N_{k},\end{cases}$

where $N_{k}$ is a threshold used to separate differing rates of deposition by collision activity. The form and coefficients, $k_{1}, k_{2}, \ldots, k_{6}$ and $N_{k}$, of Eq. (10) were determined from an investigation of collision rates in a DAMAGE study (see below).

The FADE model also includes the ability to modify the effectiveness of removal by atmospheric decay. This option 
was included following work reporting a secular decrease in atmospheric density, which would in turn increase the number of objects on orbit and the number of collisions by up to $40 \%$ (Lewis et al., 2005). The rate of change of the atmospheric decay parameter is given by,

$\dot{D}=a D$,

with typical values for $a$ being in the range $[0,-0.6] \% \mathrm{yr}^{-1}$ according to Emmert et al. (2004).

Eqs. (2)-(11) were implemented in JavaScript and embedded within a Hyper-Text Mark-up Language (HTML) document. An HTML form was used to provide users with the means to enter the initial conditions and a free graphing tool, also written in JavaScript (http://www.structura.info/
XYGraph/XYGraphDemo.htm, accessed July 2008) was used to provide real-time graph solutions for the FADE predictions. The user is able to select three graph options, the number of objects on orbit versus time, the cumulative number of collisions versus time, and the collision frequency versus time, by clicking on HTML links below the form. These links are also used to update the current graph when the initial conditions are modified. The resulting HTML page is adapted by a Content Style Sheet (CSS) file to generate the internet-based graphical user interface, shown in Fig. 2, which includes the form used to set the initial conditions and the graphical outputs. Consequently, the FADE model runs as a client-side, web-based service, with full graphical output delivered in real-time.

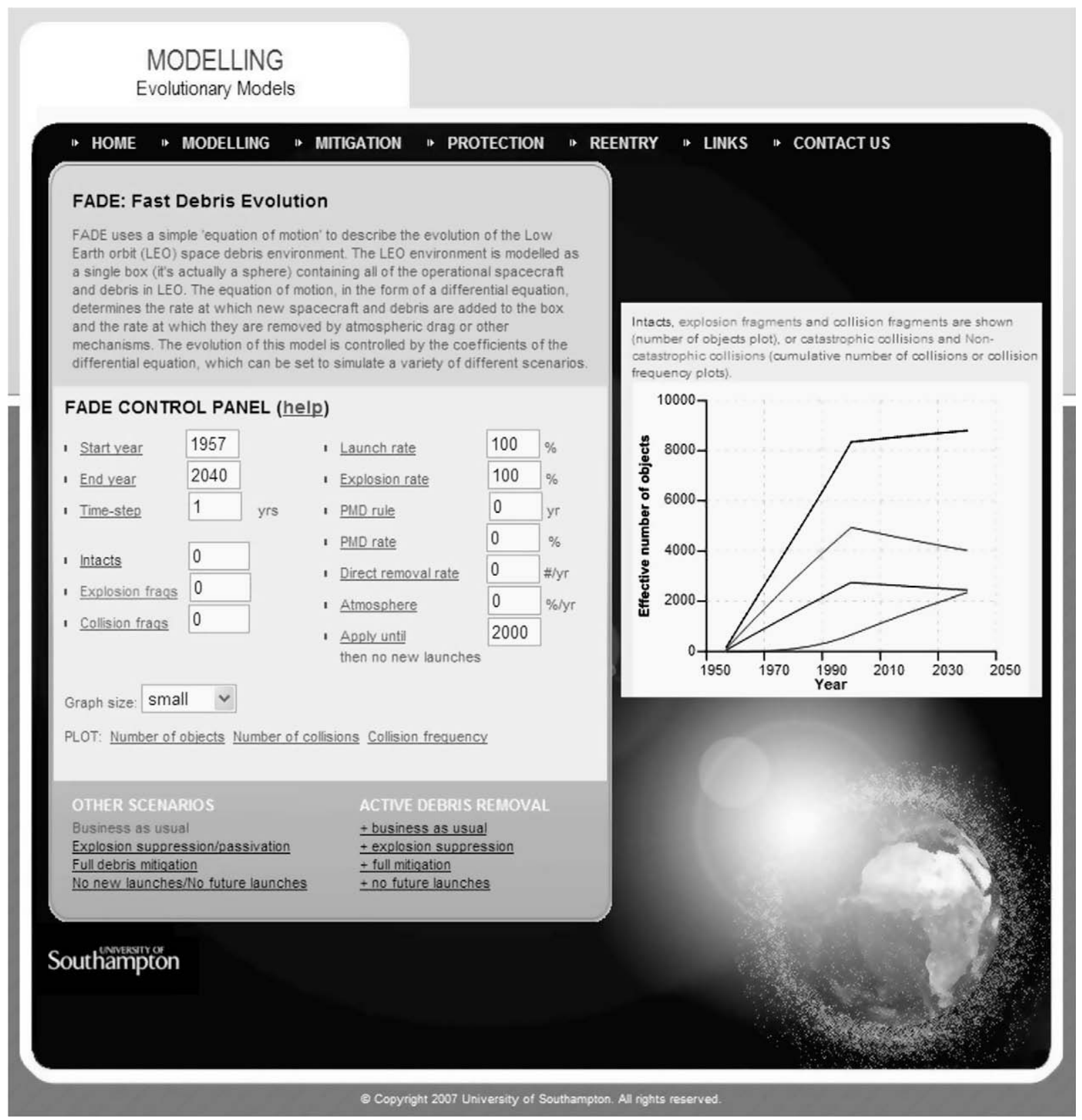

Fig. 2. FADE basic web-based user interface. 


\subsection{Choice of parameters}

The values of the parameters and coefficients in Eqs. (2)-(11) were determined by analysis of the outputs from a full MC study performed by the DAMAGE model. The study scenario, described in Table 3, aimed to show the effect of 'no new launches' on the LEO $\geqslant 10 \mathrm{~cm}$ debris environment. The projection period was 1957 through 2040, with no new launches after 1 January 2000. This particular scenario was chosen because it allowed 'deposition' and 'removal' parameters to be defined, as it featured a period of launch activity and a period where removal by atmospheric decay was the primary activity. Forty MC runs were performed with historical launch and fragmentation events simulated up to 1 January 2000 (as described above), collision probabilities estimated from 1957 onwards and fragments added to the environment when collisions were predicted. Outputs recorded for each run included the number of objects on orbit (intact objects,

Table 3

Description of the 'no new launches' after 2000 scenario simulated by DAMAGE to establish the parameters and coefficients of the FaDE model.

\begin{tabular}{ll}
\hline Parameter & Value \\
\hline Projection period & 1 October 1957-1 January 2040 \\
No new launches after & 2000 \\
Time-step & 5 days \\
Minimum object size & $10 \mathrm{~cm}$ \\
Collision prediction: cube size & $10 \mathrm{~km}$ \\
Collision prediction: active from & 1957 \\
Explosions & Only confirmed explosions of objects \\
& launched prior to 2000 allowed \\
\hline
\end{tabular}

explosion and collision fragments), the cumulative number of collisions (catastrophic and damaging), and the cumulative collision probability for the LEO volume. These data were recorded at six-monthly intervals.

Of particular interest were the time-histories of the collision probability and the number of objects, as these were used to confirm the form and the coefficients of the differential equation describing the collision rate for the LEO volume in Eq. (10). From these two data sources, the relationship between the average number of objects in LEO and the average collision probability for the volume (Fig. 3) was identified as,

$$
\dot{C}=\left\{\begin{array}{l}
4.0 \times 10^{-7} N_{T}+7.0 \times 10^{-10} N_{T}^{2}, \quad N_{T}<3500, \\
0.025-1.8 \times 10^{-5} N_{T}+4.0 \times 10^{-9} N_{T}^{2}, \quad N_{T} \geqslant 3500,
\end{array}\right.
$$

which fit the observed DAMAGE data with a correlation coefficient $R^{2}=0.955$.

Following the identification of the collision rate coefficients, the remaining FADE parameters were found using a simple, trial-and-error approach whereby the parameters were modified and 'tuned' until the state quantities (the number of intact objects, the number of explosion fragments and the number of collision fragments in LEO) predicted by FADE matched those predicted by the DAMAGE model for the 'no new launches' scenario. The tuning with DAMAGE was based on a qualitative comparison of the graphical outputs of both models rather than a rigorous quantitative assessment at this stage. This was, in part, due to the lengthy trial-and-error process but also to avoid 'over-fitting' the parameters to this 'no new launches' scenario and reducing the capacity of the

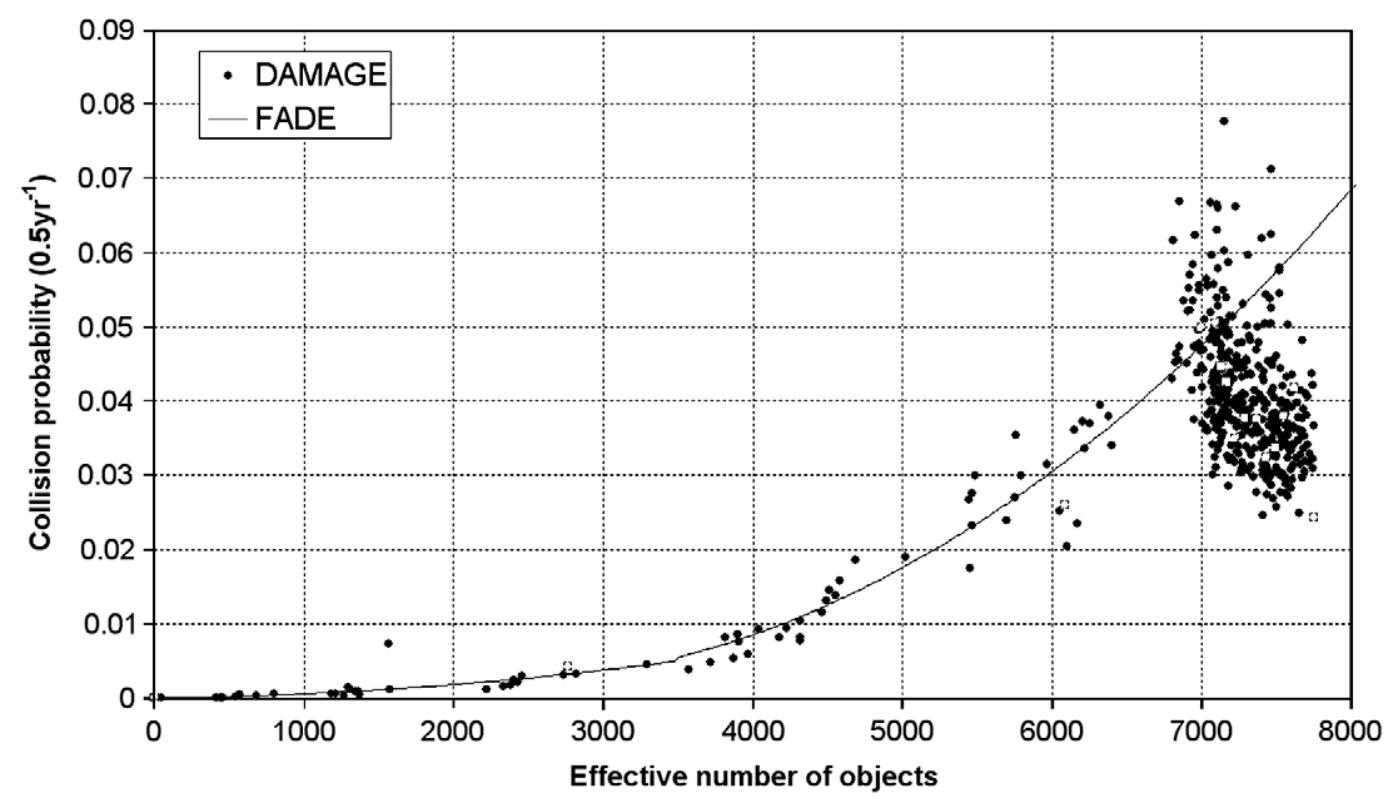

Fig. 3. The total collision probability for six-month periods as a function of the average effective number of objects $\geqslant 0 \mathrm{~cm}$ in LEO (from $40 \mathrm{MC}$ runs with DAMAGE) for the 'no new launches' after 2000 scenario (1957-2040), and the best-fit quadratic curve. 
Table 4

FADE parameters derived from the DAMAGE simulations of a 'no new launches after 2000' scenario for the projection period 1957-2040.

\begin{tabular}{ll}
\hline$a=0$ & $N_{k}=3500$ \\
$D=0.0062$ & $\dot{L}=27.5 \mathrm{yr}^{-1}$ \\
$\dot{E}=2.0 \mathrm{yr}^{-1}$ & $\dot{M}=0$ \\
$f_{c_{1}}=0.7$ & $n_{c_{1}}=625$ \\
$f_{c_{2}}=0.3$ & $n_{c_{2}}=25$ \\
$k_{1}=0$ & $n_{E}=50$ \\
$k_{2}=4.0 \times 10^{-7}$ & $n_{L}=2.75$ \\
$k_{3}=7.0 \times 10^{-10}$ & $R_{c}=0.6 \mathrm{~m}$ \\
$k_{4}=0.025$ & $R_{E}=1.0 \mathrm{~m}$ \\
$k_{5}=-1.8 \times 10^{-5}$ & $R_{I}=2.0 \mathrm{~m}$ \\
$k_{6}=4.0 \times 10^{-9}$ & $\Delta t=1$ year \\
\hline
\end{tabular}

FADE model to generalise to new scenarios. The final parameters found using this process are listed in Table 4 and the graphical outputs produced with these settings are shown in Figs. 4 and 5.

The model predictions for the number of objects in LEO show good agreement for intact objects $\left(R^{2}=0.996\right)$, explosion fragments $\left(R^{2}=0.972\right)$ and collision fragments $\left(R^{2}=0.965\right)$ through the historical 'deposition' period (1957-2000). In addition, the atmospheric decay of intact objects after the year 2000 in the FADE output shows good correspondence with the same period modelled by DAMAGE $\left(R^{2}=0.981\right)$. However, the decay of explosion fragments after the year 2000 is not captured as effectively $\left(R^{2}=0.960\right)$, due to the basic representation of atmospheric decay within the model. In the DAMAGE data, the rate of removal of explosion fragments is high initially (years 2000-2010) but lower towards the end of the projec- tion (2020-2040). Consequently, the value of $R_{E}$ was chosen to give a good fit to the removal rate at the midpoint of the 'removal' period. The correspondence between the DAMAGE and FADE predictions of the number of collision fragments after the year 2000 is good $\left(R^{2}=0.975\right)$.

Fig. 5 shows that FADE slightly underestimates the number of damaging and catastrophic collisions in the period 1957-2020, compared with the DAMAGE predictions, but then slightly overestimates the number towards the end of the projection period (2020-2040). Over the full period (1957-2040), the correlation coefficients were calculated to be $R^{2}=0.987$ for damaging collisions and $R^{2}=0.995$ for catastrophic collisions.

It is important to note that whilst the names of the parameters listed in Table 4 are associated with physical properties of the LEO environment, their values may not reflect the actual properties, being chosen purely on the basis of a match with the DAMAGE results. In fact, the values listed in Table 4 represent only one set of possible values that produce results comparable with DAMAGE. Many other combinations exist, with some that more closely match the physical properties they are attempting to represent. However, searching through all of these possible combinations to find the optimal set of parameters was not considered to be necessary for this work.

One final point to note is that because the collision rate coefficients were determined using DAMAGE data recorded at six-monthly intervals, the shortest recommended time-step, $\Delta t$ for use in the FADE model is one year as this meets the criteria for the Nyquist sampling theorem.

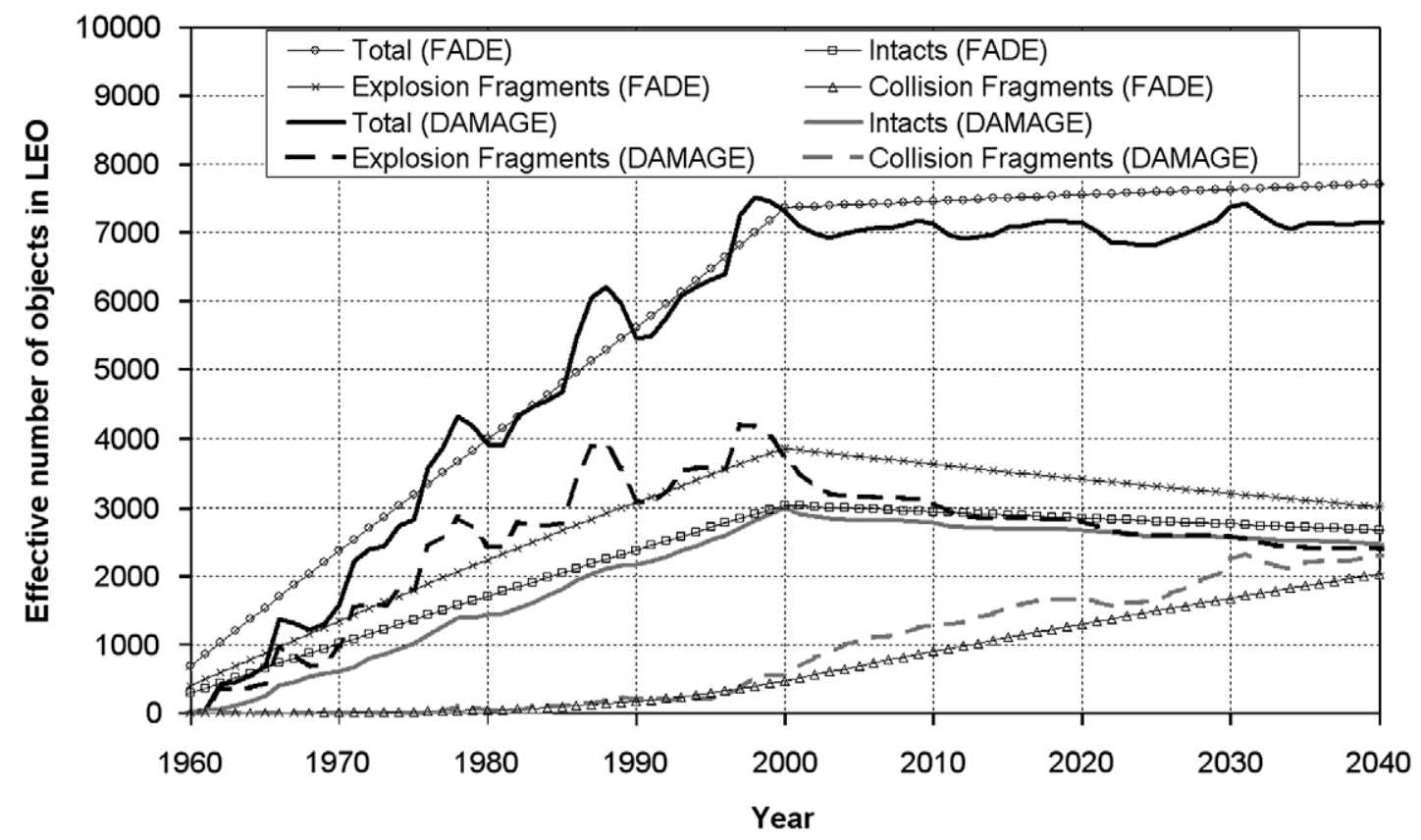

Fig. 4. Effective number of intact objects, explosion fragments and collision fragments $\geqslant 10 \mathrm{~cm}$ for the no new launches after 2000 scenario: averages from 40 DAMAGE MC runs, and FADE results. 


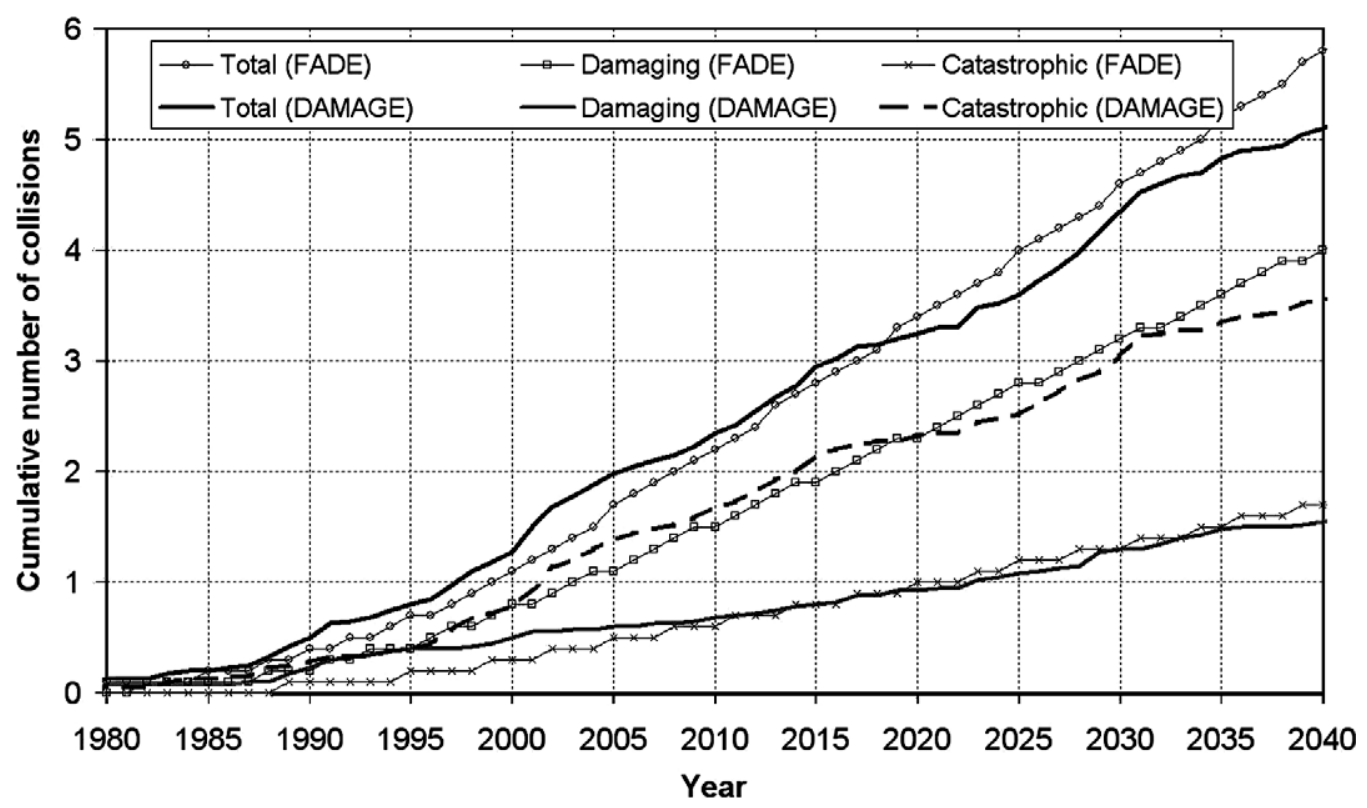

Fig. 5. Cumulative number of collisions in LEO for the no new launches after 2000 scenario: averages from 40 DAMAGE MC runs, and FADE results.

\section{Simulations}

Following the successful parameterisation of the FADE model, the ability of the code to generalise to two new scenarios is examined. Firstly, a 'business as usual' (19572040) scenario (Table 5) is used to investigate the robustness of the deposition terms in Eqs. (5)-(7) when the number of objects in LEO continues to increase. Secondly, a 'no new launches' (1957-2200) scenario (as Table 3, except for the projection period) is used to further test the removal terms in Eqs. (5)-(7) in a study that follows the work by Liou and Johnson (2006). For both scenarios, the DAMAGE model was used to check the accuracy of the results. For these 'test' cases, a simple quantitative assessment was used to determine the performance of the FADE model. This assessment was based on the differences between the FADE and DAMAGE predictions in the number of objects on orbit and the cumulative number of collisions at a survey date near the end epoch, and the correlation coefficient over the full projection period. For reasons related to the DAMAGE data export routine and the precision of the FADE outputs, the survey date chosen for the business as usual scenario was 1 January 2039 and the date

Table 5

Description of the 'business as usual' (1957-2040) scenario.

\begin{tabular}{ll}
\hline Parameter & Value \\
\hline Projection period & 1 October 1957-1 January 2040 \\
Traffic model (2000-2040) & Based on launch statistics for 1996-2001 \\
Future explosions (2000-2040) & $\begin{array}{l}\text { Based on explosion statistics for 1996- } \\
2001\end{array}$ \\
Time-step & 5 days \\
Minimum object size & $10 \mathrm{~cm}$ \\
Collision prediction: cube size & $10 \mathrm{~km}$ \\
$\begin{array}{l}\text { Collision prediction: active } \\
\quad \text { from }\end{array}$ & 1957 \\
\hline
\end{tabular}

selected for the no new launches scenario was 1 January 2199.

\section{Results}

The total number of objects predicted by FADE to be on orbit at epoch 1 January 2039 in the business as usual (1957-2040) scenario was 18,081, compared with an average of $17,320( \pm 2861)$ forecast by the DAMAGE model after $40 \mathrm{MC}$ runs. The correlation coefficient calculated over the projection period (1957-2040) was $R^{2}=0.989$. Fig. 6 shows the cumulative number of collisions, arranged by collision type, predicted by the DAMAGE and FADE models for the 'business as usual' scenario. Here, the FADE model predicted 15 collisions by 1 January 2039 compared to an average of $12.23( \pm 4.05)$ calculated from the DAMAGE MC runs, with a correlation coefficient of $R^{2}=0.985$ over the projection period.

The number of objects and the cumulative number of collisions estimated by both models for the no new launches (1957-2200) scenario are shown in Figs. 7 and 8. FADE predicted 7766 objects would be on orbit at the survey date, compared with an average of $7427( \pm 1496)$ estimated from the 40 DAMAGE MC runs, with a correlation coefficient of $R^{2}=0.986$ over the full projection period (1957-2200). The cumulative number of collisions predicted by FADE to have occurred by 1 January 2199 was 27.2, compared with $17.2( \pm 4.87)$ estimated by DAMAGE. For this parameter, the correlation coefficient over the full projection period was $R^{2}=0.996$.

\section{Discussion}

The comparison of the FADE results with those produced by DAMAGE indicated that the FADE model is 


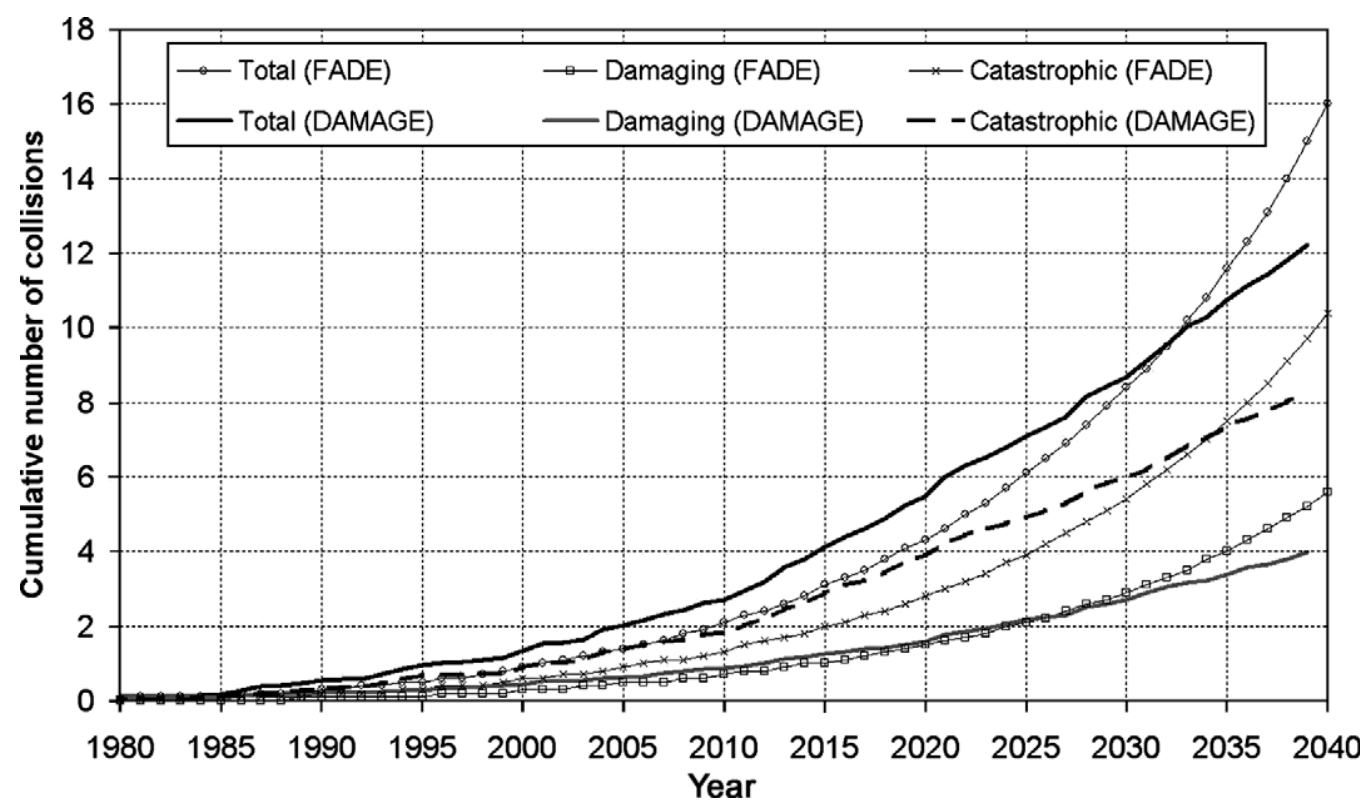

Fig. 6. Cumulative number of collisions in LEO for the business as usual scenario: averages from 40 DAMAGE MC runs, and FADE results.

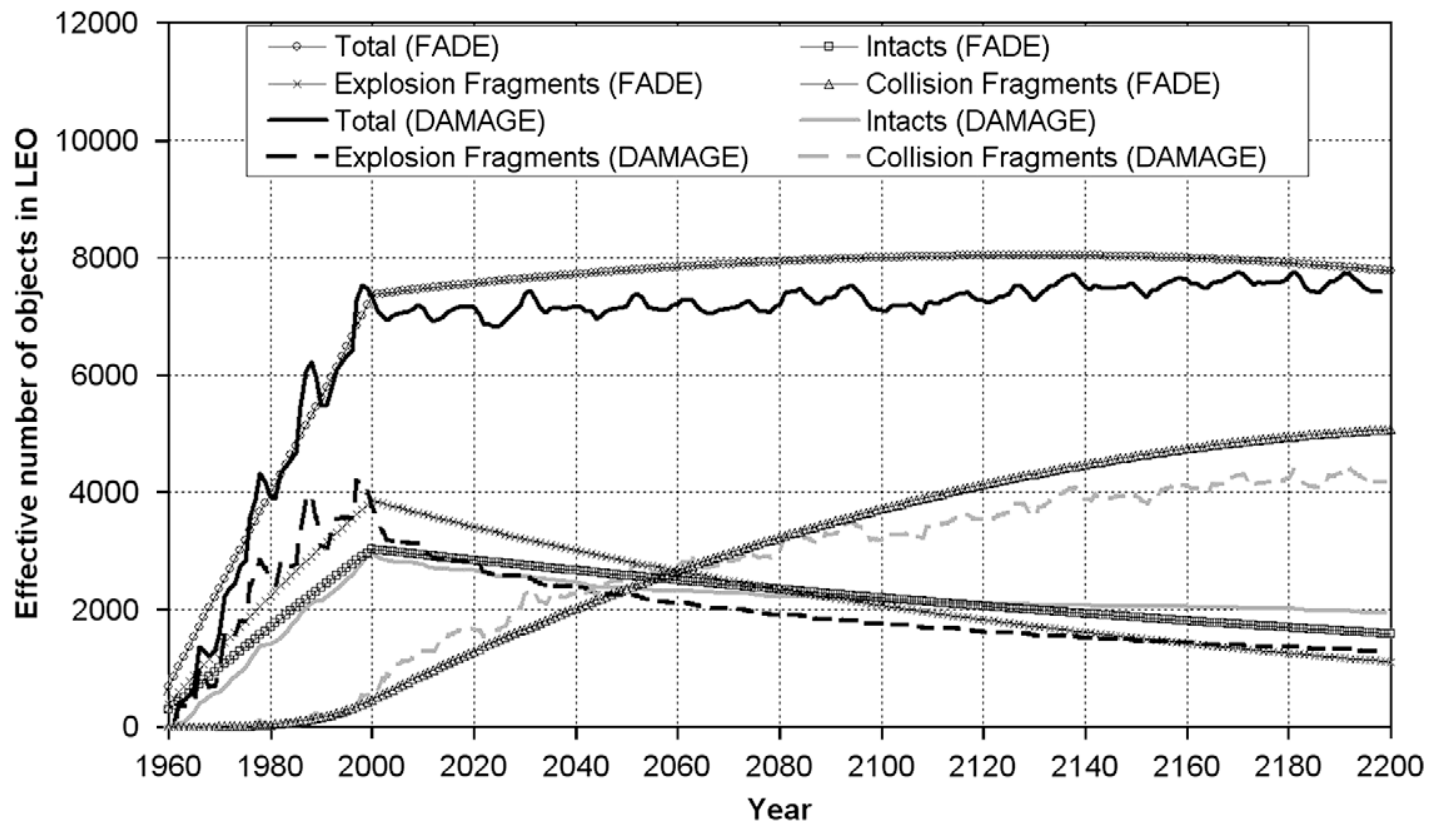

Fig. 7. Effective number of objects $\geqslant 10 \mathrm{~cm}$ in LEO for the no new launches after 2000 scenario: averages from 40 DAMAGE MC runs, and FADE results.

able to generalise to new scenarios projected into the future for several decades, in particular the 'business as usual' (1957-2040) scenario. In this scenario, the number of objects on orbit and the cumulative number of collisions predicted by FADE remained within one standard deviation of the DAMAGE averages throughout the simulation period. The similarity between the FADE and DAMAGE time-histories confirms that the form of, and the parameters used in the 'deposition' terms of Eqs. (5)-(7) are appropriate and able to capture the underlying debris source mechanisms that drive the LEO environment over medium-term projections.

The second test scenario was used to investigate the performance of the FADE model when atmospheric decay, the key debris sink mechanism, becomes the dominant force in the environment. The comparison of the two models for this 'no new launches' (1957-2200) scenario shows that the 'removal' terms in Eqs. (5)-(7) are not as successful at capturing the individual responses of the different types of debris object to atmospheric drag over longer projection 


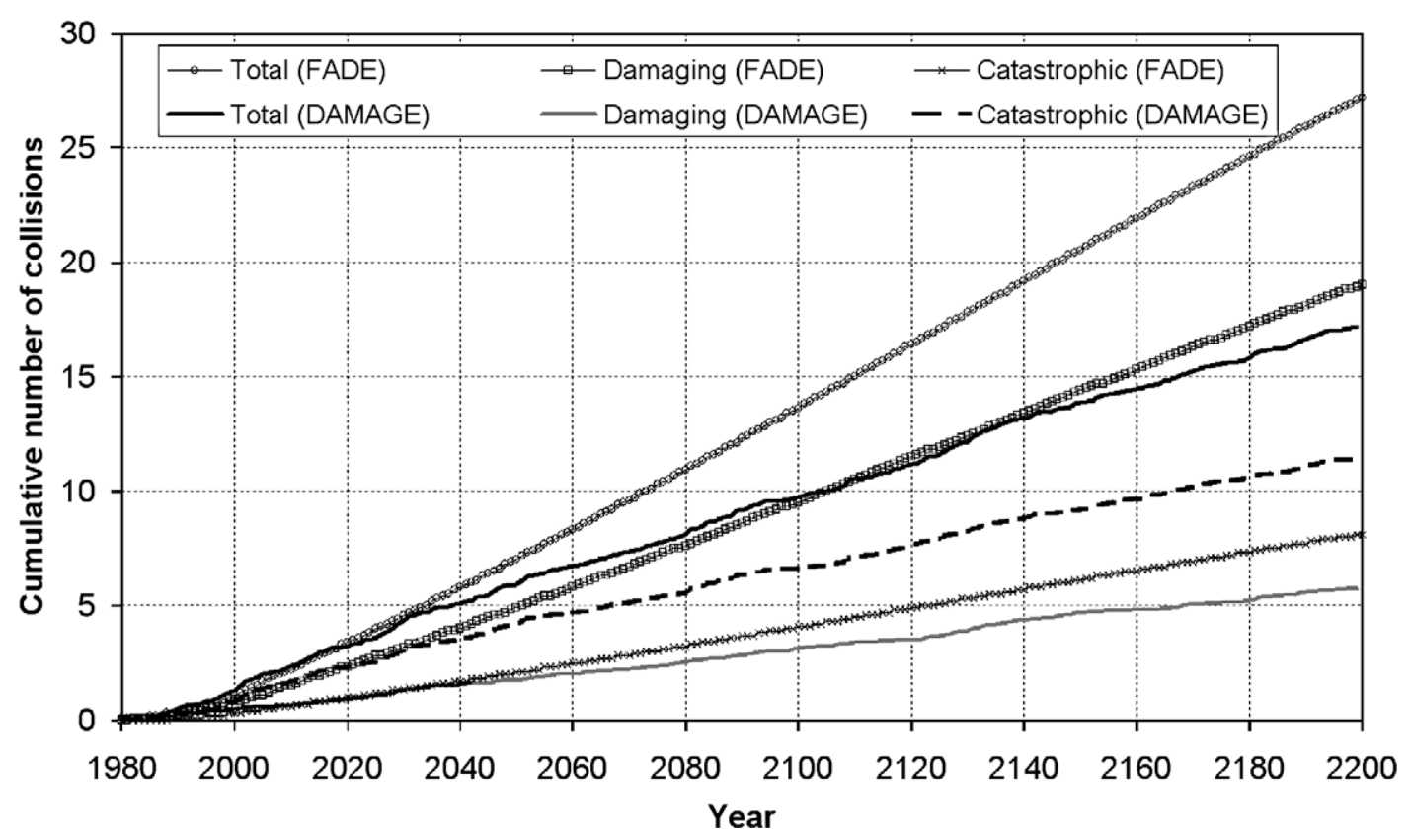

Fig. 8. Cumulative number of collisions in LEO for the no new launches after 2000 scenario: averages from 40 DAMAGE MC runs, and FADE results.

periods (beyond $80-100$ years). In addition, differences in the collision rate after the year 2040 between the DAMAGE and FADE models (Fig. 8) indicate that the coefficients relating to collisions $\left(n_{C_{1}}, n_{C_{1}}\right.$ and $\left.R_{C}\right)$ used in Table 4 are suboptimal when used to predict collision frequencies in the long term. Changes to the average radius parameters in Eqs. (5)-(7) may compensate for these differences, although these would also require nearly all the other parameters in the FADE model to be adjusted. A better approach may be to identify additional debris species (such as intact spacecraft and rocket bodies, for example), with distinct radius values, and to divide the LEO volume into altitude bins, corresponding to work reported by Talent (2008) and Rossi et al. (1994). In the Phenomenological Orbital Debris Model (PODEM; Talent, 2008), for example, 10 altitude bins and five debris species are identified, requiring 50 differential equations to model the environment. Work to develop this capability is continuing. However, given our objective to deliver a fast, flexible and transparent model via the Internet, a simplified version of this approach will be investigated initially.

The ability to produce predictions of the future debris environment that are equivalent to predictions made by complex computer codes, without the need for computationally demanding $\mathrm{MC}$ runs and in real-time, is of considerable benefit when, for example, investigating the effects of debris prevention measures. The FADE model offers users the possibility of exploring a wide range of historical and future scenarios quickly, and this capacity complements the role of the more complex codes such as DAMAGE. In many instances, using these three-dimensional models is akin to 'taking a sledgehammer to crack a nut', especially when the variables of interest describe the gross characteristics of the debris environment (e.g., the total number of objects on orbit, or the cumulative number of collisions). The PIB approach is, perhaps, more efficient in these instances, and given their precision, the complex models are better suited in investigations that require detailed information about the debris populations. An alternative point of view, however, is that the use of the FADE model forms part of a larger investigation in which high-definition, three-dimensional models are required. Supplementary information, found quickly using FADE, can be valuable when managing debris studies and it is a relatively simple task to 'tune' the FADE model to fit the outputs of debris models other than DAMAGE.

The results presented above suggest that the prototype FADE model is not sufficiently accurate in describing the details of the debris source and sink mechanisms for this approach to be used reliably for scientific research. Nevertheless, from an educational perspective the approach still offers considerable benefit to users, especially given the immediacy and simplicity of the model in its on-line form. From this perspective, the current accuracy of the FADE model is sufficient. For instance, the model is currently being used at the University of Southampton to demonstrate space debris principles and modelling issues to final year Aerospace Engineering and Physics undergraduate students, and to provide a simple modelling tool for a $\mathrm{Ph}$.D. project. In addition, FADE can also facilitate a deeper dialogue between the debris community and members of the press, with the flexibility to cover a wide range of possible scenarios using its user-friendly on-line interface.

\section{Conclusions}

This paper has introduced a new 'particles-in-a-box method, based on Talent's (1992) analytical model and 
entitled FADE, which uses a first-order differential equation to describe the evolution of the LEO debris environment. The method has been implemented as a web-based service using JavaScript, and includes a simple user interface and graphing feature for real-time exploration. The parameters used in FADE were found by fitting the model's predictions of the number of objects on orbit to similar predictions made by the University of Southampton's DAMAGE model, for a 'no new launches' scenario. Subsequently, the validity of the model and the parameters identified were tested using two new scenarios, a 'business as usual' scenario and a second 'no new launches' scenario. The results indicated that the FADE model was able to capture the key sources of debris, launch activities, explosions and accidental collisions, generating predictions of the number of objects and the cumulative number of collisions that were both within one standard deviation of those made by DAMAGE for the medium-term 'business as usual' case. However, the description of debris removal by atmospheric decay within the FADE model was found to be less accurate, with the predictions of the number of objects and the cumulative number of collisions for the second, long-term 'no new launches' scenario being outside one standard deviation determined by DAMAGE.

Further work aims to address the limitations identified in this early work, with the introduction of altitude bins and an optimisation method for identifying the FADE parameters automatically, potentially improving the model's ability to adapt to new scenarios. In the meantime, the prototype version of the FADE model will continue to be used in undergraduate teaching at the University. This version can be found at http://www.soton.ac.uk/ $\sim$ hglewis/research/debris/FADE.html.

\section{References}

Emmert, J.T., Picone, J.M., Lean, J.L., Knowles, S.H. Global change in the thermosphere: compelling evidence of a secular decrease in density. J. Geophys. Res., 109, A02301, doi:10.1029/2003JA010176, 2004.

Farinella, P., Cordelli, A. The proliferation of orbiting fragments: a simple mathematical model. Sci. Global Secur. 2, 368-378, 1991.

Johnson, N.L., Krisko, P.H., Liou, J.-C., Anz-Meador, P.D. NASA'S new breakup model of EVOLVE 4.0. Adv. Space Res. 28 (9), 1377-1384, 2001.

Kessler, D.J. Derivation of the collision probability between orbiting objects: the lifetimes of Jupiter's outer moons. Icarus 48, 39-48, 1981.

Krisko, P.H. The predicted growth of the low Earth orbit space debris environment - an assessment of future risk for spacecraft. In: Proceedings of the IMechE. Part G: J. Aerospace Engineering, vol. 221, pp. 975-985, 2007.

Lewis, H.G., Swinerd, G.G., Ellis, C.S., Martin, C.E. Response of the space debris environment to greenhouse cooling. In: Proceedings of the Fourth European Conference on Space Debris, Darmstadt, Germany, 18-20 April 2005, ESA Publication SP-587, pp. 243-248, 2005.

Liou, J.-C. A statistical analysis on the future debris environment. In: Proceedings of the 56th International Astronautical Congress, Fukuoka, Japan, October 17-21, 2005, Paper No. IAC-05-B6.2.02, 2005.

Liou, J.-C., Johnson, N.L. Risks in space from orbiting debris. Science $311,340-341,2006$.

Liou, J.-C., Johnson, N.L. A sensitivity study of the effectiveness of active debris removal in LEO. In: Proceedings of the 58th International Astronautical Congress, Hyderabad, India, Article IAC-07-A6.3.05, 2007.

Liou, J.-C., Kessler, D.J., Matney, M., Stansbery, G. A new approach to evaluate collision probabilities among asteroids, comets, and Kuiper belt objects. In: Proceedings of Lunar and Planetary Science, vol. XXXIV, 2003.

Liou, J.-C., Hall, D.T., Krisko, P.H., Opiela, J.N. LEGEND - a threedimensional LEO-to-GEO debris evolutionary model. Adv. Space Res. 34, 981-986, 2004.

Rossi, A., Cordelli, A., Farinella, P., Anslemo, L. Collisional evolution of the Earth's orbital debris cloud. J. Geophys. Res. 99, 23195-23210, 1994.

Talent, D.L. Analytical model for orbital debris environmental management. J. Spacecraft Rocket 29 (4), 508-513, 1992.

Talent, D.L. The 2007 Chinese ASAT test - implications to the impact of space warfare on the LEO environment. In: Proceedings of the 18th AAS/AIAA Space Flight Mechanics Meeting, Galveston, Texas, January 2008, 2008. 